\title{
Impact of current cough on health-related quality of life in patients with COPD
}

This article was published in the following Dove Press journal:

International Journal of COPD

13 September 2016

Number of times this article has been viewed

\section{Gaëtan Deslee,' Pierre-Régis \\ Burgel, ${ }^{2}$ Roger Escamilla, ${ }^{3}$ \\ Pascal Chanez, ${ }^{4}$ Isabelle \\ Court-Fortune, ${ }^{5}$ Pascale \\ Nesme-Meyer, ${ }^{6}$ Graziella \\ Brinchault-Rabin, ${ }^{7}$ Thierry \\ Perez, ${ }^{8}$ Gilles Jebrak, ${ }^{9}$ \\ Denis Caillaud, ${ }^{10}$ Jean-Louis \\ Paillasseur," Nicolas Roche ${ }^{2}$ \\ On behalf of the Initiatives \\ BPCO Scientific Committee}

'Department of Respiratory Diseases, INSERM UMR 903, Maison Blanche

Hospital, University Hospital of Reims,

Reims, ${ }^{2}$ Department of Respiratory

Diseases, Cochin Hospital, AP-HP and

University Paris Descartes, Sorbonne

Paris Cité, Paris, ${ }^{3}$ Department of

Respiratory Diseases, Larrey Hospital,

Toulouse, ${ }^{4}$ Department of Respiratory

Diseases, APHM, INSERM UI 077.

CNRS UMR 7733 Aix Marseille

Université, Marseille, ${ }^{5}$ Department

of Respiratory Diseases, University

Hospital of St Etienne, ${ }^{6}$ Department

of Respiratory Diseases, La Croix

Rousse Hospital, Lyon, ${ }^{7}$ Department

of Respiratory Diseases, Pontchaillou

Hospital, University Hospital of

Rennes, Rennes, ${ }^{8}$ Department of

Respiratory Diseases, Calmette

Hospital, University Hospital of Lille,

Lille, ${ }^{9}$ Department of Respiratory

Diseases, Bichat Hospital, AP-HP, Paris,

${ }^{10}$ Department of Respiratory Diseases,

Gabriel Montpied Hospital, University

Hospital of Clermont-Ferrand,

Clermont-Ferrand, "EFFI-STAT,

Paris, France

Correspondence: Gaëtan Deslee

Service de Pneumologie, INSERM U903,

Hôpital Maison Blanche-CHU de Reims,

45, rue Cognacq-Jay, 51092 Reims,

France

$\mathrm{Tel}+333267876 \mathrm{II}$

Fax +33 326788356

Email gdeslee@chu-reims.fr
Background: Cough and sputum production are frequent in chronic obstructive pulmonary disease (COPD). The objective of this study was to examine the relationship between cough and sputum production and health-related quality of life in COPD.

Methods: A cross-sectional study was conducted in the French Initiatives COPD cohort and assessed cough and sputum production within the past 7 days using the cough and sputum assessment questionnaire (CASA-Q), health-related quality of life, spirometry, smoking status, dyspnea, exacerbations, anxiety and depression, and comorbidities.

Results: One hundred and seventy-eight stable COPD patients were included (age, 62 [56-69] years, 128 male, forced expiratory volume in 1 second [ $\left.\mathrm{FEV}_{1}\right]$ : 57 [37-72] \% predicted) (median [Q1-Q3]). In univariate analyses, health-related quality of life (Saint George's respiratory questionnaire total score) was associated with each CASA-Q domain and with chronic bronchitis, exacerbations, dyspnea, $\mathrm{FEV}_{1}$, depression, and anxiety. All four domains introduced separately were independently associated with health-related quality of life. When introduced together in multivariate analyses, only the cough impact domain remained independently associated with health-related quality of life $\left(R^{2}=0.60\right)$. With chronic bronchitis (standard definition) instead of the CASA-Q, the $R^{2}$ was lower $\left(R^{2}=0.54\right)$.

Conclusion: This study provides evidence that current cough in the previous 7 days is an important determinant of health-related quality of life impairment in stable COPD patients.

Keywords: signs and symptoms, respiratory, sputum, questionnaires, dyspnea, multivariate analysis

\section{Introduction}

Cough and sputum production are frequent symptoms in chronic obstructive pulmonary disease (COPD) patients. The prevalence of chronic cough and sputum production varies from $14 \%$ to $74 \%$ in COPD patients, depending on the definitions used for chronic bronchitis, the case report forms, and the characteristics of the patients. ${ }^{1-6}$ Chronic cough and sputum production, usually defined using the classic definition of "chronic bronchitis" (cough and sputum production for at least 3 months of two consecutive years), are associated with a higher risk of exacerbation and hospitalization,,$^{1,3,4,7,8}$ faster lung function decline, ${ }^{9,10}$ mortality, ${ }^{8,11-14}$ and health-related quality of life (HRQoL) impairment. ${ }^{2,4}$ Current smoking is the main risk factor for chronic bronchitis in COPD. ${ }^{15}$ Occupational and domestic exposures to gases, fumes, and dusts are also associated with a higher prevalence of chronic bronchitis in COPD patients. ${ }^{16-18}$

Until recently, despite the high prevalence of cough and sputum production in COPD and their association with the key features of COPD, these symptoms have been overlooked in clinical research on COPD, which likely relates to the lack of effective dedicated therapeutic agent. The natural history of chronic bronchitis in COPD is 
not clearly understood, and there is a lack of dedicated and objective tools for assessing the symptoms of cough and sputum production in COPD. Dedicated questionnaires have been designed, but their utility and relationship with other COPD outcomes have not been investigated intensively.

The present cross-sectional study was conducted in a subsample of the French Initiatives COPD cohort, a cohort of stable COPD patients. Cough and sputum production within the past 7 days were assessed using the cough and sputum assessment questionnaire (CASA-Q), which contains four domain scores, assessing the symptoms and impact of cough and sputum. This dedicated questionnaire allows a better characterization and quantification of the symptoms of cough and sputum than the classic definition of "chronic bronchitis". HRQoL was assessed using the Saint George's Respiratory Questionnaire (SGRQ). The objective of this study was to test the hypothesis that current cough and sputum production in the previous 7 days assessed by a dedicated questionnaire, the CASA-Q, are associated with HRQoL impairment in COPD.

\section{Methods}

\section{Study design}

This cross-sectional multicenter study was conducted in a subsample of the observational French Initiatives bronchopneumopathie chronique obstructive cohort including 18 French university hospitals. ${ }^{1}$ The relationships between cough and sputum assessed by the CASA-Q and the HRQoL were assessed using univariate and multivariate analyses.

\section{Patient selection}

The inclusion criteria were as follows: a diagnosis of COPD defined as a postbronchodilator forced expiratory volume in 1 second $\left(\mathrm{FEV}_{1}\right) /$ forced vital capacity $<0.7$ measured in stable condition with no history of exacerbation within the four previous weeks. The exclusion criterion was a predominant diagnosis of asthma, bronchiectasis, or any other significant respiratory disease.

The study was approved by the ethics committee of Versailles, France (number: 04-479) for the protection of human beings involved in biomedical research. An informed written consent was obtained from each subject.

\section{Data collection}

Data collection has been extensively described previously. ${ }^{1}$ A standardized questionnaire was used to collect demographic data (age, sex, body mass index [BMI]), smoking history (current status and cumulative smoking), comorbidities, symptoms of dyspnea using the modified Medical Research Council (mMRC) scale, symptoms of chronic bronchitis defined as daily cough and sputum production for at least 3 months per year during the past 2 years, hospital anxiety and depression scale, and the number of exacerbations in the previous year. HRQoL was assessed using the SGRQ. Pulmonary function tests were performed according to American Thoracic Society/European Respiratory Society standards. ${ }^{19,20}$

\section{Cough and sputum assessment questionnaire}

CASA-Q was added to the standardized questionnaires in the Initiatives BPCO cohort for the purpose of this study. This questionnaire has been described in detail elsewhere. ${ }^{21,22}$ Briefly, the CASA-Q is a self-administered questionnaire that assesses cough and sputum based on their frequency, severity, and impact on daily activities in the previous 7 days. The CASA-Q contains four domains: cough symptoms, cough impact, sputum symptoms, and sputum impact. Each domain contains three to eight items, each of which is answered in five categories from "never" to "always" for frequency and from "not at all" to "a lot/extremely" for intensity. For each domain, the items are summed and rescaled to obtain a score ranging from 0 to 100 , with higher scores associated with fewer symptoms or less impact. The validated French translation of the CASA-Q was used in this study and is presented in the US English version in Table S1. ${ }^{21,22}$

\section{Statistical analysis}

Quantitative variables are expressed as median and quartiles and/or number and percentages. Univariate analyses were performed to determine the relationship between SGRQ total score and the following variables: age, sex, smoking status, cumulative smoking, $\mathrm{BMI}, \mathrm{FEV}_{1} \%$ predicted, dyspnea mMRC grade, number of exacerbations in the previous year, hospital anxiety and depression scale (cutoff $\geq 10$ ), cardiovascular comorbidities, chronic bronchitis, and CASA-Q domains. Multivariate models were built to assess the contribution of CASA-Q domains to quality of life impairment. Patients with complete data on the CASA-Q and the variables identified as being associated with the total SGRQ score in univariate analyses were included in the multivariate analyses. The first model was built with SGRQ total score as the explained variable and only the four CASA-Q domains as the possible explanatory variables (ie, no other variable was introduced in the model) (model 1). All other models also included all the variables tested with the SGRQ total score in univariate analysis as covariates $(P<0.35)$. In the 
first of these models, all CASA-Q domains were introduced (model 2). In a following series of four models, each domain was introduced separately (one model for each domain) (models 3-6). Finally, a model was built without any CASA-Q domain, but with chronic bronchitis following its standard definition (model 7).

\section{Results}

\section{Patient characteristics and CASA-Q results}

Overall, 178 patients recruited in the Initiatives BPCO cohort were assessed for cough and sputum production using the CASA-Q. Clinical characteristics of the patients are presented in Table 1.

The median values for the four domains of the CASA-Q assessing cough and sputum in the previous 7 days were 70.8 (50.0-91.7) for cough symptoms, 84.4 (59.4-100.0)

Table I Clinical characteristics of the population $(\mathrm{N}=178)$

\begin{tabular}{|c|c|c|}
\hline Variables & Missing data, $\mathbf{n}$ & $\begin{array}{l}\text { Median (Q I-Q3) } \\
\text { or n (\%) }\end{array}$ \\
\hline Age, years & 0 & $62(56-69)$ \\
\hline Male & 0 & $128(72)$ \\
\hline Smoking, pack-year & 2 & $36(24-53)$ \\
\hline Current smokers & 2 & $63(36)$ \\
\hline Body mass index & 0 & $25(22-29)$ \\
\hline Chronic bronchitis & 8 & $123(69)$ \\
\hline Exacerbations in previous year & 1 & \\
\hline 0 & & $61(34)$ \\
\hline 1 & & 51 (29) \\
\hline$>2$ & & $65(37)$ \\
\hline $\begin{array}{l}\text { Congestive heart failure and } \\
\text { coronary artery disease }\end{array}$ & 0 & $4 I(23)$ \\
\hline mMRC dyspnea scale & 4 & \\
\hline 0 & & $19(11)$ \\
\hline I & & $69(40)$ \\
\hline 2 & & $44(25)$ \\
\hline 3 & & $37(2 I)$ \\
\hline 4 & & $5(3)$ \\
\hline $\mathrm{FEV}_{1}$ & 0 & $57(37-72)$ \\
\hline Anxiety $\mathrm{HADS} \geq 10$ & 6 & $49(28)$ \\
\hline Depression HADS $\geq 10$ & 5 & $29(16)$ \\
\hline SGRQ total score & 0 & $44(29-57)$ \\
\hline \multicolumn{3}{|l|}{ CASA-Q score } \\
\hline Cough symptoms & 0 & 70.8 (50.0-91.7) \\
\hline Cough impact & I & $84.4(59.4-100.0)$ \\
\hline Sputum symptoms & 5 & $66.7(41.7-88.1)$ \\
\hline Sputum impact & 6 & $87.5(66.7-100.0)$ \\
\hline
\end{tabular}

Note: Data are presented as median (QI-Q3) or number (n) and percentage (\%). Abbreviations: CASA-Q, cough and sputum assessment questionnaire; $\mathrm{FEV}_{1}$, forced expiratory volume in I second; HADS, Hospital Anxiety Depression Scale with higher scores indicating more severe symptoms; mMRC, modified Medical Research Council dyspnea score from 0 to 4 with higher score indicating more severe dyspnea; SGRQ, Saint George's Respiratory Questionnaire ranging from 0 to 100 with lower score indicating more severe health-related quality of life impairment. for cough impact, 66.7 (41.7-88.1) for sputum symptoms, and 87.5 (66.7-100.0) for sputum impact. Chronic bronchitis defined as daily cough and sputum production for at least 3 months each year during the past 2 years was found in $69 \%$ of the patients. Each score of the four domains of the CASA-Q was significantly lower with the presence of chronic bronchitis $(P<0.0001)$, indicating more cough and sputum symptoms or impact when chronic bronchitis, based on the usual definition, was present (Figure 1).

\section{Variables associated with the SGRQ total score in univariate analyses}

Univariate analyses demonstrated that each CASA-Q domain score was associated with the total SGRQ score: cough symptoms $(R=-0.391, P<0.0001)$, cough impact $(R=-0.586$, $P<0.0001)$, sputum symptoms $(R=-0.263, P<0.0005)$, and sputum impact $(R=-0.481, P<0.0001)$. No significant association was found between the total SGRQ score and age, sex, BMI, smoking status, cumulative smoking, and cardiovascular comorbidities. Conversely, the total SGRQ score was associated with chronic bronchitis, number of exacerbations in the previous year, mMRC dyspnea grade, $\mathrm{FEV}_{1} \%$ predicted, and the hospital anxiety and depression subscales (Table 2).

\section{Independent contribution of cough and sputum production to quality of life impairment}

One hundred and forty-eight COPD patients with insightful data on the CASA-Q and the variables identified as associated with the total SGRQ score in univariate analyses were

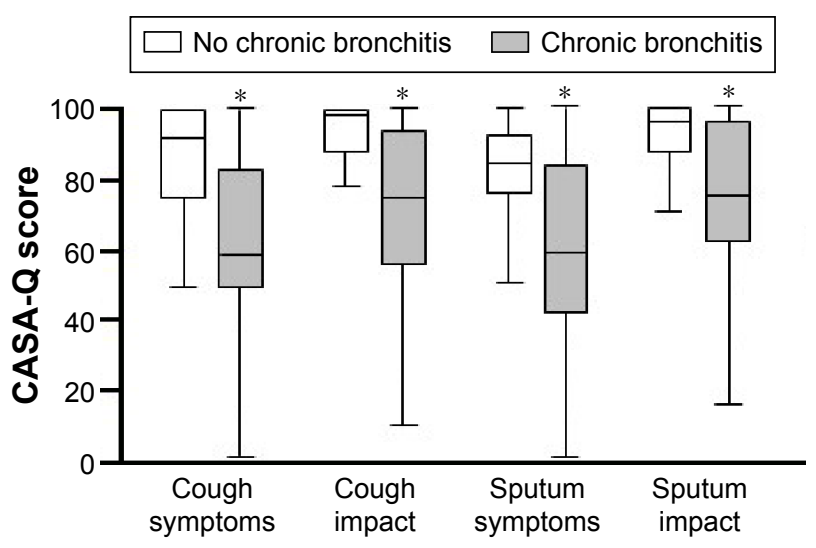

Figure I CASA-Q scores for each domain and chronic bronchitis.

Notes: The CASA-Q assessed symptoms and impact in the previous 7 days. Chronic bronchitis was defined as daily cough and sputum production for at least 3 months each year during the past 2 years. All four CASA-Q domains (cough symptoms, cough impact, sputum symptoms, and sputum impact) were lower when chronic bronchitis was present $(* P<0.0001)$, indicating more cough and sputum symptoms or impact.

Abbreviation: CASA-Q, cough and sputum assessment questionnaire. 
Table 2 Associations between the total SGRQ score and other characteristics of patients in univariate analyses

\begin{tabular}{lll}
\hline Variables & $\boldsymbol{R}$ & $\boldsymbol{P}$-value \\
\hline Age & -0.02 & 0.7876 \\
Sex & & 0.7049 \\
Smoking, current & & 0.8014 \\
Body mass index & -0.003 & 0.9586 \\
Chronic bronchitis & -0.41 & 0.0002 \\
Exacerbations (previous year) & 0.391 & $<0.000$ I \\
Cardiovascular comorbidity & & 0.3377 \\
mMRC dyspnea scale & 0.602 & $<0.0001$ \\
FEV & -0.372 & $<0.0001$ \\
Anxiety HADS $\geq 10$ & & 0.0001 \\
Depression HADS $\geq 10$ & & 0.0002 \\
CASA-Q score & & \\
$\quad$ Cough symptoms & -0.391 & $<0.0001$ \\
Cough impact & -0.586 & $<0.0001$ \\
Sputum symptoms & -0.263 & 0.0005 \\
Sputum impact & -0.481 & $<0.0001$ \\
\hline
\end{tabular}

Abbreviations: CASA-Q, cough and sputum assessment questionnaire; FEV , forced expiratory volume in I second; HADS, Hospital Anxiety Depression Scale with higher scores indicating more severe symptoms; mMRC, modified Medical Research Council dyspnea score from 0 to 4 with higher score indicating more severe dyspnea; SGRQ, Saint George's Respiratory Questionnaire ranging from 0 to 100 with lower score indicating more severe health-related quality of life impairment.

included in the multivariate analyses (Table 3 ). There were no statistically significant differences between the clinical characteristics of the 148 patients included in the multivariate analyses and the 30 patients not included because of incomplete data. When all the CASA-Q domains were considered without other variables, both the cough symptoms and cough impact scores were statistically associated with the total SGRQ score (model 1). Multivariate analyses including all CASA-Q domains, and $\mathrm{FEV}_{1} \%$ predicted, mMRC dyspnea grade, hospital anxiety and depression scale, cardiovascular comorbidities, and the number of exacerbations in the previous year as covariates retained the cough impact domain as the sole CASA-Q domain independently associated with SGRQ total score in the final model (model 2). When all the CASA-Q domains were introduced separately, they were all retained as independently associated with SGRQ total score in their specific models (models 3-6). When chronic bronchitis was introduced instead of CASA-Q domains, it was also independently associated with SGRQ total score, but the variance explained was lower than in the model with the CASA-Q cough impact domain (model 7).

\section{Discussion}

This multicenter cross-sectional study demonstrates that current cough, assessed by the dedicated CASA-Q questionnaire in the 7 previous days, is independently associated with HRQoL impairment in COPD. Importantly, the cough impact domain of the CASA-Q was a stronger determinant of HRQoL impairment than chronic bronchitis, indicating that current cough, rather than current sputum production or chronic cough and sputum production, is responsible for reduced HRQoL in COPD patients.

We used the dedicated CASA-Q questionnaire to examine the contribution of current cough and sputum production to HRQoL impairment in COPD patients. A recent study in a large population of 4,513 subjects from the COPDGene cohort demonstrated that adding answers to the questions about cough and sputum from the SGRQ to the usual definition of chronic bronchitis allowed identifying more patients with similar phenotypes associated with poorer outcomes. ${ }^{23}$ Our study concurs with the study by Kim et al in establishing new ways of assessing cough and sputum production and their contribution to important outcomes in COPD patients. Dedicated questionnaires (eg, the CASA-Q questionnaire)

Table 3 Relationships between the SGRQ total score, and CASA-Q domain scores and clinical variables in multivariate analyses

\begin{tabular}{|c|c|c|c|c|c|c|c|}
\hline Candidate variables & Model I & Model 2 & Model 3 & Model 4 & Model 5 & Model 6 & Model 7 \\
\hline CASA-Q cough impact & $<0.0001$ & $<0.0001$ & $<0.000 \mathrm{I}$ & & & & \\
\hline CASA-Q cough symptoms & 0.0435 & NS & & 0.00012 & & & \\
\hline CASA-Q sputum impact & 0.0581 & NS & & & $<0.0001$ & & \\
\hline CASA-Q sputum symptoms & NS & NS & & & & 0.0020 & \\
\hline $\mathrm{FEV}_{1}, \%$ & & 0.0058 & 0.0024 & 0.00019 & 0.0072 & 0.0068 & 0.0042 \\
\hline mMRC dyspnea scale & & $<0.0001$ & $<0.0001$ & $<0.0001$ & $<0.0001$ & $<0.0001$ & $<0.0001$ \\
\hline Exacerbations & & 0.0334 & 0.0266 & 0.0028 & 0.0320 & 0.0046 & 0.0037 \\
\hline Depression & & 0.0558 & 0.0229 & 0.0037 & 0.0338 & 0.0080 & 0.0017 \\
\hline Anxiety & & 0.0889 & 0.0995 & 0.0331 & 0.0542 & 0.0169 & 0.0090 \\
\hline Cardiovascular comorbidities & & 0.0072 & 0.00043 & 0.0019 & 0.0167 & 0.0101 & 0.0068 \\
\hline Chronic bronchitis & & & & & & & 0.0498 \\
\hline$R^{2}$ & 0.38 & 0.60 & 0.62 & 0.56 & 0.58 & 0.54 & 0.54 \\
\hline
\end{tabular}

Abbreviations: CASA-Q, cough and sputum assessment questionnaire; FEV , forced expiratory volume in I second; mMRC, modified Medical Research Council dyspnea score from 0 to 4 with higher score indicating more severe dyspnea; NS, not significant; SGRQ, Saint George's Respiratory Questionnaire ranging from 0 to I00 with lower score indicating more severe health-related quality of life impairment. 
and/or cough monitors ${ }^{15}$ could represent useful tools for assessing these symptoms and their contribution to patientreported outcomes in clinical trials and real life.

In the present study, the CASA-Q scores of each domain demonstrated significant association with HRQoL impairment. The correlation coefficients were relatively weak in univariate analyses, except for the cough impact domain which exhibited a stronger correlation coefficient. Interestingly, there was a large variability of the CASA-Q scores of each domain, even when chronic bronchitis was present. Chronic cough is often assessed by simple questions on daily or usual cough and sputum production, eventually completed by data related to chronology parameters such as date of onset and duration (at least 3 months each year during the past 2 years). In the present study, each domain score of the CASA-Q was strongly lower when chronic bronchitis was present, but multivariate analyses demonstrated that the association of HRQoL impairment was greater with the cough impact domain score than with the usual definition of chronic bronchitis. These results demonstrate that a dedicated questionnaire assessing cough in the previous 7 days is more accurate to identify cough-associated HRQoL impairment in COPD than the usual definition of chronic bronchitis assessing cough and sputum production during the past 2 years.

The CASA-Q targets cough and sputum by an original approach assessing both the symptoms and impacts in daily activities within the last 7 days. ${ }^{21}$ A study assessing the evolution of the CASA-Q domains' scores during and after COPD exacerbation demonstrated the responsiveness of the CASA-Q to clinical recovery after COPD exacerbation, ${ }^{22}$ suggesting its usefulness for monitoring cough and sputum production symptoms and their impacts in longitudinal studies. Interestingly, the feasibility of using the CASA-Q for assessing cough and sputum was also demonstrated in idiopathic pulmonary fibrosis. ${ }^{24}$ Our study confirmed this feasibility in a large group of COPD subjects included in a multicenter cohort. It should be pointed out that the diagnosis of COPD in the Initiative BPCO cohort is secured on international criteria using a standard definition of COPD and standardized questionnaires, allowing the collection of prospective data on demographics, symptoms, smoking history, and comorbidities. ${ }^{1}$

One strength of this study is the strategy of multivariate analyses including data on all domains of the CASA-Q together with the major factors associated with poor outcomes in COPD, allowing to thoroughly characterize the contribution of cough to HRQoL impairment. When all variables were considered in the multivariate analyses, the CASA-Q cough impact score was found to be significantly associated with HRQoL impairment, compared to dyspnea assessed by the
mMRC scale. The other variables significantly associated in the multivariate analyses were $\mathrm{FEV}_{1}$, exacerbations, depression, anxiety, and cardiovascular comorbidities. It should be noted that the $R^{2}$ values of the different models of the multivariate analyses were not much different (0.54-0.62), indicating that each CASA-Q domain score remained pertinent for its association with HRQoL impairment. These results suggest that assessing cough is as important as assessing other key elements associated with poor outcomes and used to guide therapeutic choices in COPD, such as dyspnea, $\mathrm{FEV}_{1}$, and exacerbations. Accordingly, the CASA-Q could be of great interest to efficiently assess cough and sputum in COPD for better characterization of COPD phenotypes and to assess the response to innovative treatments in clinical trials.

\section{Limitations}

One limitation is the cross-sectional nature of the study, which prevents from linking CASA-Q data with longitudinally collected outcomes. The sample size is also a potential limitation; indeed, the generalizability of these results should be tested in larger populations. The 7-day period assessed with the CASA-Q does not provide information regarding the impact of cough and sputum symptoms on HRQoL in a shorter or longer than 7-day period. It would be interesting to investigate the relationships between the CASA-Q scores and the COPD assessment test questionnaire that includes eight items with two items assessing cough and sputum production and which is used in routine clinical practice. It could be argued that cough and sputum production are likely to relate to HRQoL as measured by the SGRQ, since this questionnaire actually comprises questions on cough and sputum production. However, there are only two questions on these symptoms in the SGRQ; so, their contribution to the total score is minimal. In addition, a sensitivity analysis was performed to assess the relationship between the CASA-Q and the total score of the SGRQ calculated after removing the two questions on cough and sputum production; identical results were found (data not shown).

\section{Conclusion}

This study provides evidence that current cough in the previous 7 days is an important determinant of HRQoL impairment in stable COPD patients and suggests that the dedicated questionnaire CASA-Q is an interesting tool to monitor cough and sputum production in COPD clinical trials.

\section{Acknowledgment}

The French Initiative BPCO cohort is supported by an unrestricted grant from Boehringer Ingelheim and Pfizer, which had no role in the study design. 


\section{Author contributions}

GD, P-RB, J-LP, and NR have made substantial contributions to the conception or design of the work. GD, P-RB, RE, PC, IC-F, PN-M, GB-R, TP, GJ, DC, J-LP, and NR contributed to acquisition, analysis, and interpretation of data for the work. GD, P-RB, J-LP, and NR drafted the work. GD, P-RB, RE, PC, IC-F, PN-M, GB-R, TP, GJ, DC, J-LP, and NR revised the article critically for important intellectual content. GD, P-RB, RE, PC, IC-F, PN-M, GB-R, TP, GJ, DC, J-LP, and NR gave their final approval of the version to be published. All authors contributed toward data analysis, drafting and revising the paper and agree to be accountable for all aspects of the work.

\section{Disclosure}

GD reports collecting personal fees from Boehringer Ingelheim, AstraZeneca, Novartis, Chiesi, and BTG/ PneumRx; P-RB reports personal fees from Boehringer Ingelheim, Astra Zeneca, Novartis, GSK, and Vertex; RE reports personal fees from Boehringer Ingelheim, Novartis, AstraZeneca, Mundipharma, ALK, and Chiesi; PC reports personal fees from Almirall, Boehringer Ingelheim, Centocor, GSK, MSD, AstraZeneca, Novartis, Teva, Chiesi, and Schering Plough; IC-F reports personal fees from Boehringer Ingelheim, Novartis, and Pfizer; PN-M reports personal fees from Boehringer Ingelheim; GB-R reports personal fees from Boehringer Ingelheim, Novartis, GSK, Chiesi, and Pfizer; TP reports personal fees from Boehringer Ingelheim, Novartis, GSK, Chiesi, and Pierre Fabre; GJ reports personal fees from Boehringer Ingelheim, Pfizer, Novartis, and GSK; DC reports personal fees from Boehringer Ingelheim and Roche; J-LP reports no conflict of interest; NR reports personal fees from Boehringer Ingelheim, Novartis, Teva, GSK, AstraZeneca, Chiesi, Mundipharma, Cipla, and Pfizer. The authors report no other conflicts of interest in this work.

\section{References}

1. Burgel PR, Nesme-Meyer P, Chanez P, et al. Cough and sputum production are associated with frequent exacerbations and hospitalizations in COPD subjects. Chest. 2009;135(4):975-982.

2. Agusti A, Calverley PM, Celli B, et al; Evaluation of COPD Longitudinally to Identify Predictive Surrogate Endpoints (ECLIPSE) investigators. Characterisation of COPD heterogeneity in the ECLIPSE cohort. Respir Res. 2010;11:122.

3. de Oca MM, Halbert RJ, Lopez MV, et al. The chronic bronchitis phenotype in subjects with and without COPD: the PLATINO study. Eur Respir J. 2012;40(1):28-36.

4. Kim V, Davey A, Comellas AP, et al; COPDGene ${ }^{\circledR}$ Investigators. Clinical and computed tomographic predictors of chronic bronchitis in COPD: a cross sectional analysis of the COPDGene study. Respir Res. 2014;15:52.

5. Miravitlles M, Guerrero T, Mayordomo C, Sánchez-Agudo L, Nicolau F, Segú JL. Factors associated with increased risk of exacerbation and hospital admission in a cohort of ambulatory COPD patients: a multiple logistic regression analysis. The EOLO Study Group. Respiration. 2000; 67(5):495-501.
6. Kim V, Criner GJ. The chronic bronchitis phenotype in chronic obstructive pulmonary disease: features and implications. Curr Opin Pulm Med. 2015;21(2):133-141.

7. Corhay JL, Vincken W, Schlesser M, Bossuyt P, Imschoot J. Chronic bronchitis in COPD patients is associated with increased risk of exacerbations: a cross-sectional multicentre study. Int J Clin Pract. 2013; 67(12):1294-1301.

8. Lindberg A, Sawalha S, Hedman L, Larsson LG, Lundbäck B, Rönmark E. Subjects with COPD and productive cough have an increased risk for exacerbations and death. Respir Med. 2015;109(1):88-95.

9. Vestbo J, Prescott E, Lange P. Association of chronic mucus hypersecretion with FEV1 decline and chronic obstructive pulmonary disease morbidity. Copenhagen City Heart Study Group. Am J Respir Crit Care Med. 1996;153(5):1530-1535.

10. Camiciottoli G, Bigazzi F, Paoletti M, Cestelli L, Lavorini F, Pistolesi M. Pulmonary function and sputum characteristics predict computed tomography phenotype and severity of COPD. Eur Respir J. 2013; 42(3):626-635

11. Annesi I, Kauffmann F. Is respiratory mucus hypersecretion really an innocent disorder? A 22-year mortality survey of 1,061 working men. Am Rev Respir Dis. 1986;134(4):688-693.

12. Guerra S, Sherrill DL, Venker C, Ceccato CM, Halonen M, Martinez FD. Chronic bronchitis before age 50 years predicts incident airflow limitation and mortality risk. Thorax. 2009;64(10):894-900.

13. Pelkonen M, Notkola IL, Nissinen A, Tukiainen H, Koskela H. Thirtyyear cumulative incidence of chronic bronchitis and COPD in relation to 30-year pulmonary function and 40-year mortality: a follow-up in middle-aged rural men. Chest. 2006;130(4):1129-1137.

14. Lange P, Nyboe J, Appleyard M, Jensen G, Schnohr P. Relation of ventilatory impairment and of chronic mucus hypersecretion to mortality from obstructive lung disease and from all causes. Thorax. 1990;45(8): 579-585.

15. Sumner H, Woodcock A, Kolsum U, et al. Predictors of objective cough frequency in chronic obstructive pulmonary disease. Am J Respir Crit Care Med. 2013;187(9):943-949.

16. Rodríguez E, Ferrer J, Zock JP, et al; PAC-COPD Study Group. Lifetime occupational exposure to dusts, gases and fumes is associated with bronchitis symptoms and higher diffusion capacity in COPD patients. PLoS One. 2014;9(2):e88426.

17. Dijkstra AE, de Jong K, Boezen HM, et al. Risk factors for chronic mucus hypersecretion in individuals with and without COPD: influence of smoking and job exposure on CMH. Occup Environ Med. 2014;71(5): 346-352.

18. Marchetti N, Garshick E, Kinney GL, et al; COPDGene Investigators. Association between occupational exposure and lung function, respiratory symptoms, and high-resolution computed tomography imaging in COPDGene. Am J Respir Crit Care Med. 2014;190(7):756-762.

19. Quanjer PH, Tammeling GJ, Cotes JE, Pedersen OF, Peslin R, Yernault JC. Lung volumes and forced ventilatory flows. Eur Respir J. 1993; 6(Suppl 16):5-40.

20. Miller MR, Hankinson J, Brusasco V, et al; ATS/ERS Task Force. Standardisation of spirometry. Eur Respir J. 2005;26(2):319-338.

21. Crawford B, Monz B, Hohlfeld J, et al. Development and validation of a cough and sputum assessment questionnaire. Respir Med. 2008; 102(11):1545-1555.

22. Monz BU, Sachs P, McDonald J, Crawford B, Nivens MC, Tetzlaff K. Responsiveness of the cough and sputum assessment questionnaire in exacerbations of COPD and chronic bronchitis. Respir Med. 2010;104(4): 534-541.

23. Kim V, Crapo J, Zhao H, et al; COPDGene Investigators. Comparison between an alternative and the classic definition of chronic bronchitis in COPDGene. Ann Am Thorac Soc. 2015;12(3):332-339.

24. Gries KS, Esser D, Wiklund I. Content validity of CASA-Q cough domains and UCSD-SOBQ for use in patients with idiopathic pulmonary fibrosis. Glob J Health Sci. 2013;165(6):131-141. 


\section{Supplementary material}

Table SI Cough and sputum assessment questionnaire (CASA-Q)

\section{Question}

Over the last 7 days:

I. How much did you cough when you woke up in the morning?

2. How often did you cough during the day?

3. How often did you have coughing bouts?

4. How often were you tired after coughing?

5. How often did coughing make you short of breath?

6. How annoyed were you by your cough?

7. How often did you avoid going to public places because of your cough (eg, movie theaters, restaurants, etc)?

8. How often were your usual activities interrupted by your cough (eg, driving, hobbies, working around the house)?

9. How often did your cough interrupt your conversations with others (eg, phone conversations and face-to-face)?

10. How often did your cough wake you up, prevent you from falling asleep or falling back to sleep?

II. How often were you uncomfortable about bothering other people while coughing?

12. How thick was your phlegm?

13. How often did you bring up phlegm?

14. How often did your phlegm make it difficult for you to breathe?

15. How difficult was it for you to bring up phlegm?

16. How often did you feel uncomfortable about bothering other people while bringing up phlegm?

17. How annoyed were you by your phlegm?

18. How often did your phlegm interfere with your ability to speak?

19. How often did your phlegm prevent you from going to public places (eg, movie theaters, restaurants, etc)?

20. How often did you have to interrupt your usual activities to get rid of your phlegm (eg, driving, hobbies, working around the house)?

Note: Each item is answered ranging from "never" to "always" or from "not at all" to "a lot/extremely", as applicable; each type is answered using five categories.

International Journal of COPD

\section{Publish your work in this journal}

The International Journal of COPD is an international, peer-reviewed journal of therapeutics and pharmacology focusing on concise rapid reporting of clinical studies and reviews in COPD. Special focus is given to the pathophysiological processes underlying the disease, intervention programs, patient focused education, and self management protocols.

\section{Dovepress}

Submit your manuscript here: http://www.dovepress.com/international-journal-of-chronic-obstructive-pulmonary-disease-journal

This journal is indexed on PubMed Central, MedLine and CAS. The manuscript management system is completely online and includes a very quick and fair peer-review system, which is all easy to use. Visit $\mathrm{http}: / / \mathrm{www}$. dovepress.com/testimonials.php to read real quotes from published authors. 